\title{
HIGHLIGHTS
}

PANCREATIC CANCER

\section{High stromal expression of a-smooth-muscle actin correlates with aggressive pancreatic cancer biology}

"Quantification of $\alpha$-smooth-muscle actin ( $\alpha$-SMA) messenger RNA (mRNA) expression levels in formalin-fixed, paraffin-embedded tumor samples is useful to predict the prognosis of patients with pancreatic ductal adenocarcinoma (PDAC) after pancreatectomy," says Masao Tanaka of Kyushu University, Japan. His team's report also highlights a potential role for stroma-derived factors as a therapeutic target in this cancer.

PDAC is a highly aggressive malignancy with a prominent desmoplastic stroma, which is thought to promote tumor invasiveness, proliferation and metastasis. This stroma probably develops as a result of activation of pancreatic stellate cells (PSCs), which are present in high numbers in this cancer. "The tumor microenvironment, including tumor-stromal interactions, might enhance the aggressive nature of PDAC," comments Tanaka.

Expression of $\alpha$-SMA, a marker of activated PSCs, is increased in the stroma

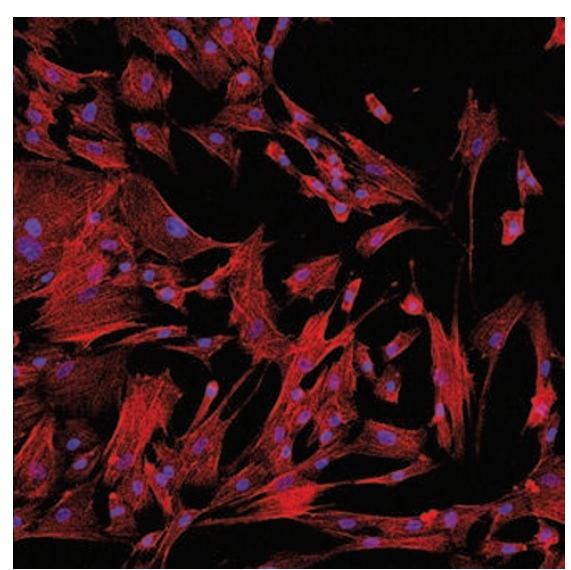

Immunofluorescent staining of $\alpha$-SMA (red) and DAPI (blue) in pancreatic stellate cells. Courtesy of M. Tanaka.

of PDACs. The researchers, therefore, measured $\alpha$-SMA mRNA expression in tumor samples from 109 patients with PDAC. The patients were divided into two similar-sized groups according to the amount of $\alpha$-SMA mRNA detected. The group with high $\alpha$-SMA mRNA expression had significantly worse survival than the group with low levels of a-SMA mRNA (5-year survival 13.6\% versus $32.3 \%$, respectively). Furthermore, a-SMA-positive activated PSCs, when co-cultivated with PDAC cells, enhanced the invasiveness and proliferation of the cancer cells. The results indicate a role for PSCs in tumor progression.

"The identification of factors derived from a-SMA-positive PSCs and clarification of their mechanisms of action are the subjects of ongoing investigations, and may lead to the development of novel therapeutic strategies directed at the PDAC microenvironment," concludes Tanaka.

Shreeya Nanda

Original article Fujita, H. et al. a-Smooth muscle actin expressing stroma promotes an aggressive tumor biology in pancreatic ductal adenocarcinoma. Pancreas 39, 1254-1262 (2010) 